\title{
FTTCNF- Novel Approach for Fault Tolerant Topology Control in Manet
}

\author{
Dr.D.Manohari $^{a}$, Dr.R.Sabitha ${ }^{b}$, Dr.B.Chellaprabha ${ }^{c}$, Dr.J.Shanthini ${ }^{d}$, Dr.M.S.Kavitha ${ }^{e}$
}

a

Associate Professor, St. Joseph's Institute of Technology, Chennai

${ }^{\mathbf{b}}$ Assistant Professor, Department of CSE, Karunya Institute of Technology and Sciences, Coimbatore - 641114,Tamilndu

cProfessor, Department of CSE, Karpagam Institute of Technology, Coimbatore-

641032, Tamilnadu

dAssociate Professor, Department of CSE, SRM Institute of Science and Technology, Kattankulathur-603203, Tamilnadu.

eAssistant Professor (SG), Department of CSE, SNS College of Technology, Coimbatore - 641035, Tamilnadu

Article History: Received: 11 January 2021; Accepted: 27 February 2021; Published online: 5 April 2021

\begin{abstract}
In wireless networks it is observed that as nodes move unpredictably sudden link disconnections occur during transmission. This leads to frequent path changes and multiple path discoveries which subsequently increase transmission of control packets in network. The nodes in the network simply rebroadcast the received route request (RREQ) packet if they do not have the route to the required destination. In addition to this, frequent hello messages for neighbour set construction and maintenance also increase control message count in the network causing a flooding issue. In order to mitigate these problems, the proposed Fault Tolerant Topology Control Neuro Fuzzy method (FTTCNF), incorporates measures to improve the network stability and to reduce the control packets in the network. These measure 1.reduce control message transmissions among neighbours by finding a stable path 2 . neighbour node distance is computed based on the reception of a signal strength Indication (RSSI), 3. path stability is decided by the link expiry time (LET) which can be used to predict the neighbour mobility deviations. These factors, ( above mentioned distance, path stability factor PSF, and LET) are subjected to the fuzzification process to identify the fuzzy rule values and are given as input to the neuron formation stage. Final neuron value is computed for all available paths and the maximum value path is chosen for data transmission. Energy level monitoring is also applied at each node to check the node's current energy and should it go below the energy threshold level the node by itself, joining the routing process is avoided. Simulation results have proved that the proposed method significantly reduces the routing overhead and improves the stability of path during data transmission.
\end{abstract}

Keywords: Hello message reduction, Receiving signal strength indication, Mobility prediction, Link and node failure, Path stability factor, Link expiry time, Control overhead

\section{Introduction}

Adhoc networks form a decentralized free movable, self-communicating node set in a network. The ultimate dispute of an adhoc network is protocol design, which needs to use minimum control packets so that the packet delivery ratio can be increased. Few on-demand routing protocols such as Adhoc On demand Distance Vector Routing (AODV) and Dynamic Source Routing (DSR) [20], have been developed for wireless adhoc communication. However, these protocols at the time of route discovery and neighbor maintenance, spend a lot of control packets. Additionally, if link disconnection occurs, a route error message will be generated by the previous node to inform source about current link disconnection status. In the intervening time, the source node initiates the new path discovery process by broadcasting RREQ again increasing the control overhead counts and subsequently reducing the packet delivery ratio (PDR). The other drawback is that end to end delay is increased from source to destination. Therefore, neighbour set management and stable path maintenance are the fundamental problems in mobile adhoc networks.

The conservative on demand flooding protocols broadcast a RREQ packet into the network to discover a route. As RREQ is broadcasted too much of rebroadcasting takes place especially in the dense deployment network area. Thus, minimizing the control packets is essential in adhoc networks.

The proposed method accomplishes the reduction in number of rebroadcasts by updating the fresh neighbors using RSSI based distance estimation. It gives a perfect dimension of the node in terms of the clarity of the listen signal from neighbours. This RSSI measurement determines the wireless channel required to maintain fresh neighbor information in each node.

Additionally, each node will require the computation energy and communication energy used during each communication. If a node looses its entire energy, it needs to quit from the network as an expiry node to avoid hole formation in routing path. Hence, periodically, nodes have to check their current energy level and if this is below the energy threshold, the node itself keeps away from forwarding the RREQ packet to establish the path. This scenario is depicted in the following fig 1. 


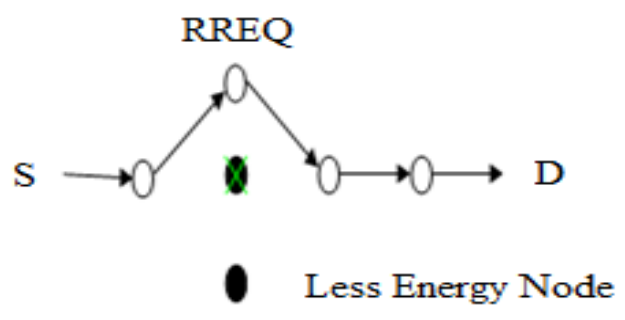

Fig 1 - Expiry Node

As a result, the nodes whose energy level is below the threshold value will not be included in routing table. Therefore, frequent link disconnections and communication failures can be avoided by this removal of expiry nodes. In mobile ad hoc network, nodes can move freely without any restriction from one place to other, making it very difficult to determine the route changes, link expiry time and node expiry. In short durations, nodes need to forecast their distance with neighbours, speed deviations, coverage area changes in order to get the link expiry time. Energy threshold monitoring and LET estimation provides strong path stability for source to destination transmissions.

Hybrid computation [19] is exploited in this work by combining the connectionist approach and fuzzy logic. Hybrid neuro fuzzy system is composed of input and output layers, and three hidden layers that represent membership functions and fuzzy rules. The first step in hybrid computation is to obtain the fuzzy rule [1] on the basis of which the fuzzy value tuning of the neural network boundary can be done. These output values are given as input to the neuron formation and the end result of neuron maximum path is chosen to deliver the data packets. The hybrid intelligent system is composed of processes as shown in the figure 2. The processing element of a hybrid neural net is called fuzzy neuron.

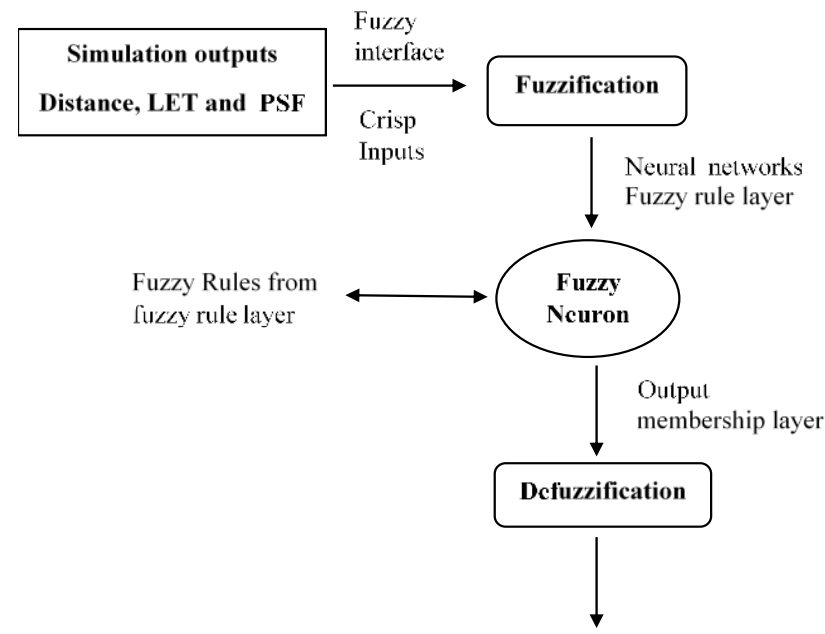

Fig 2. Hybrid Neuro fuzzy system

All these functionalities are tested with omnidirectional antenna and directional antenna model [18]. Using omnidirectional antenna the nodes are able to receive and transmit signals in all directions. Moreover, directivity and gain are associated with a directional antenna.

The salient features of this proposed method are stated as follows:

Control message broadcasting among neighbours can be reduced. Each node computes its next hop node distance using RSSI. Path stability is checked using mobility prediction of each neighbour and path stability factor can be estimated using Link Expiry Time LET [21]. Topology maintenance among local nodes without using RERR message to the source is also taken into consideration. Energy level of each neighbour node can be monitored to avoid link failure [17] and neuro fuzzy estimation output is used to establish path. This strong path construction eliminates frequent link disconnections which in turn minimize control packet broadcasting in network [3]. 
The network revamp plan expresses the significance of maintaining a connected network with less overhead even in large networks. Meanwhile, deployment of wireless nodes in a network focuses on the transmission range, neighbour's connectivity and mobility. The rest of this paper is organized as follows: Section 2 explains related existing work to date. Section 3 details of proposed method used in this work. Section 4 explains the simulation parameters and result analysis and finally, section 5 gives the conclusion summing up the features of the proposed method.

\section{Related works}

This section includes an exhaustive survey done on the Mobile Ad Hoc Network (MANET), distinguished characteristics of traditional wired networks, wireless ad hoc networks, types of ad hoc networks as well as all the existing ad hoc protocols [11]. When certain amount of data is required to be transmitted using a MANET, some data is lost due to the handoffs and/or link breakages. To avoid this loss of data, a secure link should be formed which must survive till the completion of transmission with a given definite rate of data provided by the network. The authors [21] used link expiration time (LET) as a measure in their research work representing the time until an uninterrupted link. The mobility-sensitive topology control method extends many mobilityinsensitive protocols. This method is based on two mechanisms: local view consistency based on (partially) synchronous and asynchronous "Hello" messages and buffer created by slightly increasing the actual transmission range. These two mechanisms ensure the connectivity of both logical topology and effective topology in dynamic networks [9]. This paper focuses on GPS location information and in the protocol, GPS position and velocity information are piggybacked on data packets during a live connection which is used to estimate the stability of the link between two adjacent nodes. Further, Mobile ad hoc networks have many uncertainties due to dynamic topologies, dynamic traffic and different application contexts[22]. As a well-recognized decision making technique, Fuzzy logic offers a natural way of representing and reasoning the problems with uncertainty and imprecision. [12] The neuro-fuzzy approach, symbiotically combining the merits of connectionist and fuzzy approaches, constitutes a key component of soft computing at this stage. Review of literature todate reveals that there has been no detailed and integrated categorization of the different neuro-fuzzy models used for rule generation [2] to estimate the future distance after two nodes leave each other and to evaluate the link quality when the two nodes are close to each other and the sum of every hop distance of the previous route to estimate the distance of node pairs discussed in this paper [14]. Hence, in this paper, the problem of energy efficient routing to increase the lifetime of the network is addressed.

A novel secure neighbor selection technique using recurrent reward-based learning is proposed[23] that inherits the benefits of conventional routing and intelligent machine learning paradigm for classifying the states of the nodes based on their communication behavior.

Authors in[25] aims to enhance on-demand source routing protocols by proposing two mechanisms, a zonebased route discovery mechanism (ZRDM) and a link failure prediction mechanism (LFPM). ZRDM aims to control the flooding of route requests, and LFPM aims to avoid route breakages caused by node mobility.

Authors in[26] present a new fitness function (FFn) used in the Genetic Algorithm (GA) to obtain the optimized route with a routing protocol titled as AOMDV with FFn (AOMDV-FFn). They integrated the AOMDV mechanism with the genetic algorithm (AOMDV-GA). These protocols provide an optimization process to select the efficient routes that have the highest fitness values implementing the shortest route, maximum residual energy, and less data traffic even if a random loss of data packets happens.

Mobile hosts today are powered by batterymaking efficient utilization of battery energy a very important criteria. The energy resources of actively participating nodes get depleted faster than other nodes which in some cases may lead to partitioning of the network thereby decreasing the lifetime of the network. For this reason, reduction of energy consumption is an important issue in ad hoc wireless networks [17,24].

\section{Fault tolerance topology control protocol - FTTCNF}

\subsection{Neighbour set creation}

When node $\mathbf{n}_{\mathbf{i}}$ receives a HELLO packet from its neighbors $\mathbf{n}_{\mathbf{j}}$ with its node ID and transmission time, node $\mathbf{n}_{\mathbf{i}}$ creates the neighbour list with received information. Node $\mathbf{n}_{\mathbf{i}}$ also broadcasts a HELLO packet with its ID and broadcast time. All receiving $\mathbf{n}_{\mathbf{j}}$ neighbours update $\mathbf{n}_{\mathbf{i}}$ as their neighbour in their neighbour list. Finally, all the nodes in the network construct a 1 hop neighbour list such that nodes in entire network come under connectivity.

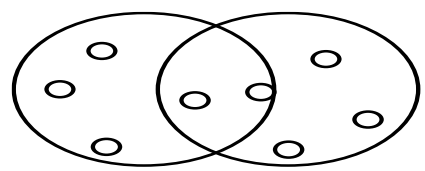

\section{Fig 3 - One-hop Neighbor set construction}




\subsection{Route Discovery}

The Network is deployed as a bound for graph $G=\{S, E\}$, where $S=\left\{s_{1}, s_{2}, \ldots . s_{n}\right\} S$ defines the set of nodes in the network and set of neighbors defined as $E, E=\left\{e_{1}, e_{2} \ldots \ldots e_{3}, e_{4}, \ldots \ldots . e_{n}\right\}$. Data transmission is initiated by source node $S$. At beginning due to on-demand nature, $S$ does not have any route to the destination to send its data packets. So the source node sends a RREQ packet with a sequence number and it is broadcasted through the wireless channel. All the nodes with highest number of neighbours under the coverage of the source receives RREQ. Limited rebroadcast will take place until RREQ reaches the destination. When RREQ reaches the physical layer (PL) of each node, PL captures the packet and computes path stability factor (PSF).

$$
\text { PSF }=1-\left(\frac{\text { Carrier sense thresh }}{\text { RSSI }}\right)^{0.25} \text {--- (1) }
$$

Here node coverage of Carrier sense thresh is chosen from the node parameters of node deployment configurations. The parameter values depend on the network environment and node configuration. After the computation of PSF, RREQ has been sent to the upper layer of the node. When a packet reaches the routing layer, distance estimation with sender and current node is done by using propagation layer. Let $S$ and receiver $j$ have coordinate values $(X 1, Y 1)$ and $(X 2, Y 2)$ respectively as node deployment coordinates. Node s and $j$ are at positions mentioned above with and have distance $\left(d_{s j}\right)$ between the nodes which is given by

$$
\left(d_{s j}\right)=\sqrt{|X 1-x 2|^{2}|Y 1-Y 2|^{2}}
$$

As the distance from (2) does not give accurate power utilization of the node for transmission, RSSI based distance estimation is used in this work. This provides accuracy of actual power used with respect to distance. RSSI is estimated when a node receives packets from previous node. The node that receives a packet starts to compute transmission power $T x P r$ of the signal of previous node and receiving node power $R x P r$ based on the current distance of receiving node from the previous node.

$$
\begin{array}{cc}
\mathrm{RSSI}=\sqrt{\frac{\left(P t \times \lambda^{2}\right)}{(4 \times \pi)^{2} * L \times P r}} & \operatorname{Pr}=R x P r \\
P t=T x P r &
\end{array}
$$

Here, $\operatorname{Pr}$ and $P t$ refers to the receiving power node and transmission power of node respectively. $L$ is considered as system loss and $\lambda^{2}$ is the wavelength of the signal. The two parameters PSF and distance $(d)$ both are estimated for complete path and the average value of these parameters are computed by the division by Hopcount taken from source to destination.

$$
\text { value }=p 1 * \frac{d}{\text { hop count }}+p 2 * \frac{P S F}{\text { hop count }}
$$

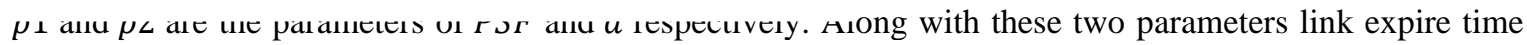
(LET) is estimated from the neighbor distance variation (nbexpire) time as third parameter $p 3$.

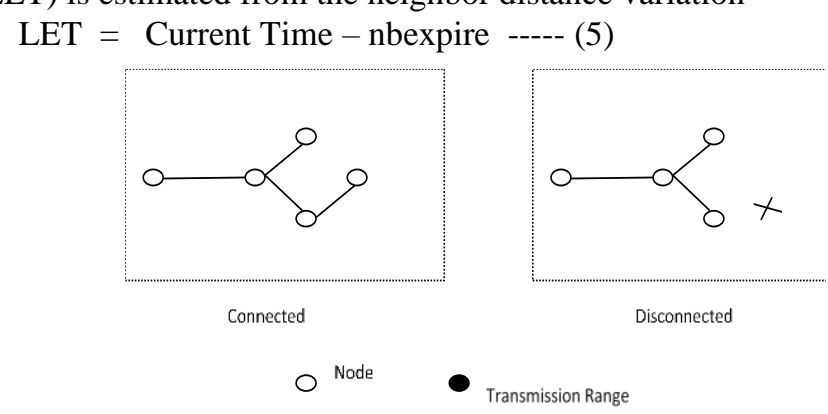

Fig 4 Link expiration

\subsection{Fuzzy estimation}

The growth of fuzzy logic was forced in big Computation to get a consistent frame which can address the concerns of indecision and lexical thought. Any reasonable logical method can be given as fuzzy input at fuzzification. Fuzzy output can be seen as the route of propagation of flexible limitation. During fuzzification 
PSF, distance and link expire time are given as linguistic variables. These inputs are given to the fuzzification process by comparing the boundary range low, medium and high values. The fuzzified inputs are used to identify the current fuzzy rules and their performance can be adjusted by tuning the rules by various combinations of low, medium and high inputs as shown in table 1.

Table 1: Rule Table

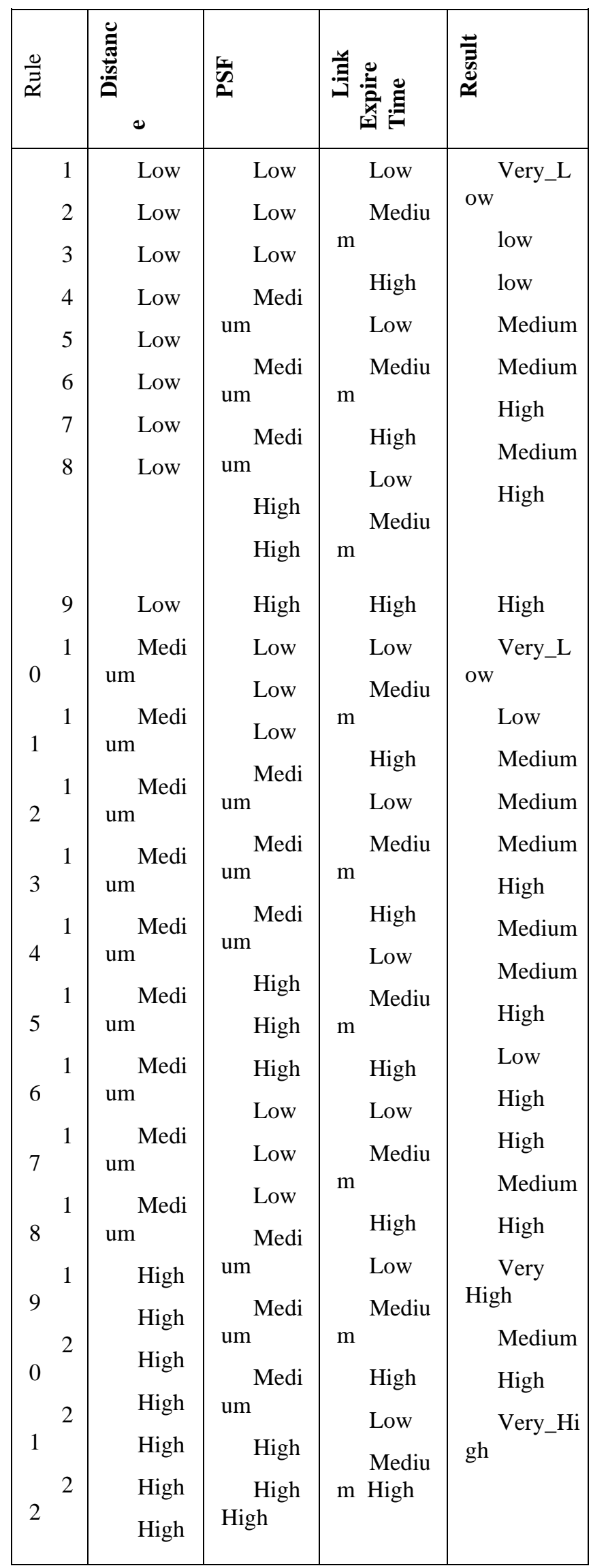




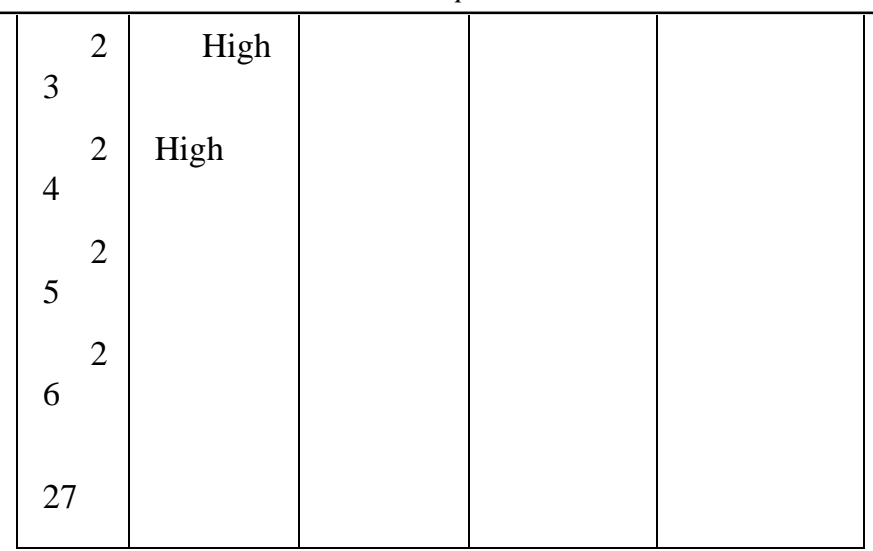

Identified fuzzy rules and the values are given as input to the neuron formation stage. Input value and computed fuzzy set values are combined to identify the neuron input parameter. Based on the membership functions the following calculations are done:

If the rule is very low (VLOW) or LOW, then neuron value is estimated as

$$
\text { nsmall }=1-2 \times \text { value }
$$

Where value is taken from eqn 4

If it is medium, then

$$
\text { nmedium }=1-2 \times \text { abs }\left(\left(\frac{\text { value }-1}{2}\right)\right)
$$

If it is high or very high neuron then

$$
\text { nhuge }=2 \times(\text { value }-1)
$$

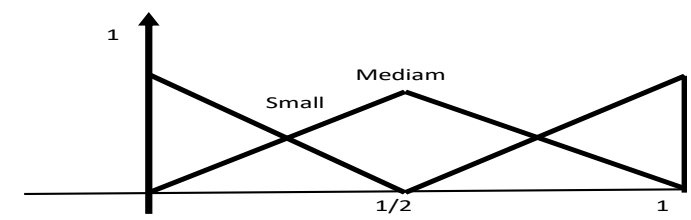

Fig 4 - Membership Functions-I

These 3 parameters applied for the fuzzification process by comparing the boundary range are rated as low, medium, high values. The training set is estimated from this rule as If $n s m a l l$ neuron value, output is considered as $-v e$ or else if nmedium value output is about to 0 . In case it shows nhuge then output is +ve.

$\{($ nsmall, $-v e),($ nmedium, about to 0$)$,

The neuron signal value $X j$ and $\mathrm{Yj}$

$$
(\text { nhuge, }+\mathrm{ve})\}
$$

is estimated as follows.

$$
\begin{array}{r}
X j=\frac{(j-1)}{4} \\
Y j=-1+\frac{(i-1)}{2}----(10)
\end{array}
$$

The values of $j$ and $i$ are between (1,5 ) random value. The distribution function is defined as inverse of summation of unit with negative exponential of each signal and is computed for both $X j$ and $Y j$. Neuron utilize transfer function $f$ is used to compute its output.

$$
\begin{aligned}
f_{x} & =\frac{1}{(1+\exp (-X j))} \\
f_{y} & =\frac{1}{(1+\exp (-Y j))}
\end{aligned}
$$

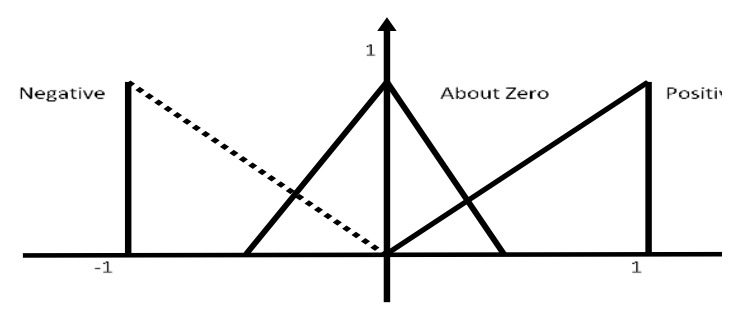

Fig 5 Membership functions - II 
Three input parameters are aggregated by the triangular corner for 3 values. These values are computed in two formats as weighted sum and direct sum of input parameters. The state the inputs which are usually membership degrees of a fuzzy concept is $i 1, i 2, i 3$ and the weights $w 1, w 2, w 3$ which has a value in the range $[0,1]$.

Sum $=\mathrm{i} 1 * \mathrm{w} 1+\mathrm{i} 2 * \mathrm{w} 2+\mathrm{i} 3 * \mathrm{w} 3=\mathrm{i} 1+\mathrm{i} 2+\mathrm{i} 3-\cdots$

The output of the system according to discrete center of gravity defuzzification method is given by

Output $=\frac{\text { sum }}{\alpha}$

Where $\alpha=\mathrm{i} 1+\mathrm{i} 2+\mathrm{i} 3$

These processes are completed in the first layer of hybrid network. If the output of the system is -ve and nonzero value then the next layer is executed. In the second layer, each level neuron is identified in firing level.

$\frac{1}{(1+\exp (\text { input } *(\alpha-i n p u t)))}$

In the third layer, each neuron is normalized in firing level by dividing the sum of each neuron.

Sum $_{a}=a 1+a 2+a 3$

Where

$$
\begin{aligned}
a 1 & =\frac{1}{1+\exp (i 1 *(\alpha-i 1))} \\
a 2 & =\frac{1}{1+\exp (i 2 *(\alpha-i 2))}
\end{aligned}
$$

The normalized values are calculated as follows

$$
a 3=\frac{1}{1+\exp (i 3 *(\alpha-i 3))}
$$

$$
\begin{aligned}
B 1 & =\frac{a 1}{(\text { sum_a })} \\
B 2 & =\frac{a 2}{(\text { sum_a })} \\
B 3 & =\frac{a 3}{(\text { sum_a }}
\end{aligned}
$$

From all incoming signals, a pattern is formed and its measurement error is computed.

$E k=\frac{1}{2 *\left(f_{y}-o k\right) *\left(f_{y}-o k\right)}---(17)$

Here ok - is the computed output

$f_{y}$ - is the actual output

The normalized neuron aggregation is done with a triangular corner and the weighted sum value is computed for each neuron as follows:

Sum $=b 1 * w 1+b 2 * w 2+b 3 * w 3 \quad---(18)$

The final neuron value is computed as the product of the weighted sum, error value and output of membership function.

$$
\text { final_param }=\text { abs }\left(\text { final_param } * E_{-} \_1 * E_{-} k 2 * \text { param }\right)
$$

where

param - may be nsmall, nmedium and nhuge from the Equations 6 to 8 and E_k1 \& E_k2 are error values calculated according to Equation 17. For all available paths, the final neuron value is computed and the maximum value path is selected for the data transmission by the destination node and the route is sent via the selected best path.

\section{Simulation Parameters and Performance Analysis}

\subsection{Implementation}

This Proposed protocol is a modified form of the base code of AODV in Network Simulator NS-2. FTTCNF protocol broadcasts one hop Hello packets to find the neighbours in sequence. To construct the complete list of neighbours RSSI value is computed to find the neighbours from which a powerful signal is received. This RSSI value depends on the characteristics of wireless medium. LET is also computed to know the link disconnection period to get accurate PSF. To extend the network lifetime, each node computes its current energy level with given energy threshold. If it is lower than given energy threshold the node itself rejects forwarding RREQ. Thus the goal is to restrict the lower energy node to participate in the active routing path. In addition, using neuro fuzzy computation complex problem of path selection is solved. FTTCNF constructs linguistic rules building a fuzzy inference set. Data from simulation is the training data for the neural network. The Overall procedure of FTTCNF is as depicted below

\section{Algorithm FTTCNF( )}

\{ Input : PSF, distance and LET 
// Average value of distance $(d) \& P S F(p)$ of entire path(d/hop-count, p/ hop-count)

// LET $(l)$ - of the link between previous and destination node

// Calculate value using distance and PSF of entire path for the first RREQ

$/ /$ value $=p 1 *$ distance $/$ hop-count $+p 2 * P S F /$ hop-count)

// p1\&p2 are random values for distance and PSF respectively

Output : stable route

For each neighbor node e $\epsilon \mathrm{E}$

// Fuzzification is done for the parameters

If $(\mathrm{d}<\mathrm{D}$ _MIN) return LOW

else if $(\mathrm{d}>=$ D_MIN \&\& d < = D_MAX)

return MED;

else if( $d>$ D_MAX)

return $\mathrm{HIGH}$;

// Similarly linguistic labels are assigned for PSF and LET by comparing the

respective MAX and MIN thresholds.

// Compare the fuzzified values of distance, PSF and LET with Linguistic label range

// Fuzzy Rule generation

$$
\begin{aligned}
& \text { if }(\mathrm{fd}==\mathrm{LOW} \& \& \mathrm{fp}==\mathrm{LOW} \& \& \mathrm{fl}==\mathrm{LOW}) \\
& \text { return VLOW; } \\
& \text { if }(\mathrm{fd}==\mathrm{LOW} \& \& \mathrm{fp}==\text { LOW \& \& fl == MED }) \\
& \text { return LOW; } \\
& \text { if }(\mathrm{fd}==\mathrm{LOW} \& \& \mathrm{fp}==\mathrm{LOW} \& \& \mathrm{fl}==\mathrm{HI}) \\
& \text { return LOW; }
\end{aligned}
$$

// Similarly all 27 possible combinations are compared

// Finally Fuzzy neuron formation has been done

// Route with max neuron value is chosen.

\subsection{Simulation Environment}

In order to evaluate the performance of the FTTCNF, a comparison was done with basic AODV and Estimated Geometrical distance routing EGD in NS-2. Network parameters were mentioned as the Distributed Coordination Function (DCF) of the 802.11 wireless MAC layer protocol used with 2Mbps bit rate radio channel model and 250 meters node coverage area was assigned as its transmission range. In application layer constant bit rate (CBR) was chosen as data packet transmission. Because of the ad-hoc nature, any node could send and receive data of size 2000 bytes per second. Random way point mobility model was applied in this simulation. Every node moves in random direction with random speed from [1, maximum speed] given and it stops in a particular place as its temporary stop point up to the pause-time of 5 seconds then chooses a new stop point and speed. Network mobility was set at 5meters /second. The total simulation time was set at 200 seconds with $1000 \mathrm{X} 1000$ network area. The various simulation parameters used are given in Table 2.

Table 2 Simulation Parameters

\begin{tabular}{|l|l|}
\hline val $(\mathrm{x})$ val $(\mathrm{y})$ & $1000 \mathrm{X} 1000 \mathrm{sqm}$ \\
\hline No. of nodes & 50 to 250 \\
\hline Total simulation time & 200.0 seconds \\
\hline Initial Energy & 100 Joules \\
\hline Transmission power & $0.02 \mathrm{Mw}$ \\
\hline Receiving power & $0.01 \mathrm{Mw}$ \\
\hline MAC & $802 \_11$ \\
\hline Antenna & Omni/Directional Antenna \\
\hline Packet size & 2000 bytes \\
\hline CST(coverage) & $3.652 \mathrm{e}-10 \quad \# 250$ \\
\hline
\end{tabular}

The performance analysis was done by i) varying the number of nodes ii) varying the speed of mobile nodes and iii) data rate. All the three scenarios were done for both directional and omni-directional antenna.

\subsection{Performance Metrics}

The Metrics that were used to evaluate the performance of FTTCNF are as listed below.

Control Overhead - It is the total number of routing packets required for network communication. 
Energy consumption is the total energy consumed in the network for the transmission of all the packets from source to destination.

Packet delivery ratio is defined as the number of data packets delivered to the destination to the number of packets generated by the CBR traffic source.

$$
\text { PDR }(\%)=\frac{\text { TotalDeliveredPackets }}{\text { TotalSentPackets }} X 100
$$

End to- End delay

End-to-end delay or one-way delay (OWD) refers to the time taken for a packet to be transmitted across a network from source to destination.

Throughput

Network throughput is the amount of data moved successfully from one place to another in a given time period.

\subsection{Results}

\subsubsection{Result analysis based on number of nodes}

In this section, the evaluation was done by varying the number of nodes from 50 to 250. Table 3 and Figures 6.a) to 6.c) show the performance of FTTCNF, AODV and EGD for the metrics control overhead, residual energy and average energy consumption respectively. The values in the table show that control overhead for FTTCNF is less than that of EGD and AODV. For directional antenna control overhead of FTTCNF is $61.99 \%$ less than EGD and $68.44 \%$ less that of AODV. Control over head for EGD and AODV is increased as the number of nodes is increased. This is because RREQ reaches dead end at some moments ie) non-availability of neighbouring nodes to forward the request in EGD and in AODV redundant rebroadcast of RREQ increases the control overhead. FTTCNF preserves greater residual energy than EGD by $0.04 \%$ and by $0.25 \%$ greater than AODV. With respect to energy consumption, FTTCNF consumes lesser energy than EGD by $48.21 \%$ and by $84.65 \%$ than AODV. The proposed FTTCNF reduces overhead which results in lesser energy consumption in nodes and greater residual energy.

Table 3Performance Evaluation FTTCNF, EGD \& AODV Dense Node distribution - Directional Antenna

\begin{tabular}{|c|c|c|c|c|c|c|c|c|c|}
\hline \multirow[b]{2}{*}{ 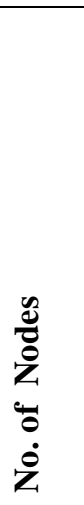 } & \multicolumn{3}{|c|}{$\begin{array}{l}\text { Control overhead } \\
\text { (in bytes) }\end{array}$} & \multicolumn{3}{|c|}{$\begin{array}{l}\text { Residual Energy } \\
\text { (in Joules) }\end{array}$} & \multicolumn{2}{|c|}{$\begin{array}{c}\text { Average } \\
\text { consumption } \\
\text { (in watts) }\end{array}$} & Energy \\
\hline & 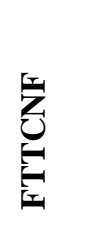 & 烏 & 光 & 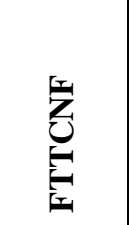 & Ð & â & 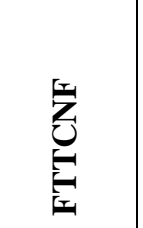 & 고도 & 光 \\
\hline 50 & 5638 & $20^{140}$ & $\begin{array}{ll} & 1947 \\
4 & \end{array}$ & \begin{tabular}{ll|} 
& 99.9 \\
2 &
\end{tabular} & 99.83 & 99.61 & $4^{0.082}$ & $2 \begin{array}{ll} & 0.165 \\
2 & \end{array}$ & 0.3935 \\
\hline 100 & 10357 & $35^{229}$ & $8^{2658}$ & $\begin{array}{ll} & 99.9 \\
7 & \end{array}$ & 99.93 & 99.84 & $3 \quad 0.030$ & $2 \quad 0.065$ & 0.1555 \\
\hline 150 & 15317 & $29^{379}$ & $3 \begin{array}{ll}3999 \\
3 & \end{array}$ & \begin{tabular}{ll|} 
& 99.9 \\
7 & \\
\end{tabular} & 99.95 & 99.81 & $6^{0.026}$ & $2 \quad 0.049$ & 0.1859 \\
\hline 200 & 20553 & $78^{647}$ & $\begin{array}{ll} & 7989 \\
7 & \end{array}$ & 699.9 & 99.92 & 99.68 & $6^{0.041}$ & $2 \quad 0.078$ & 0.3198 \\
\hline 250 & 25503 & $33^{748}$ & 19860 & $6^{99.9}$ & 99.93 & 99.59 & $5 \quad 0.037$ & $\begin{array}{ll} & 0.067 \\
7 & \end{array}$ & 0.4146 \\
\hline
\end{tabular}




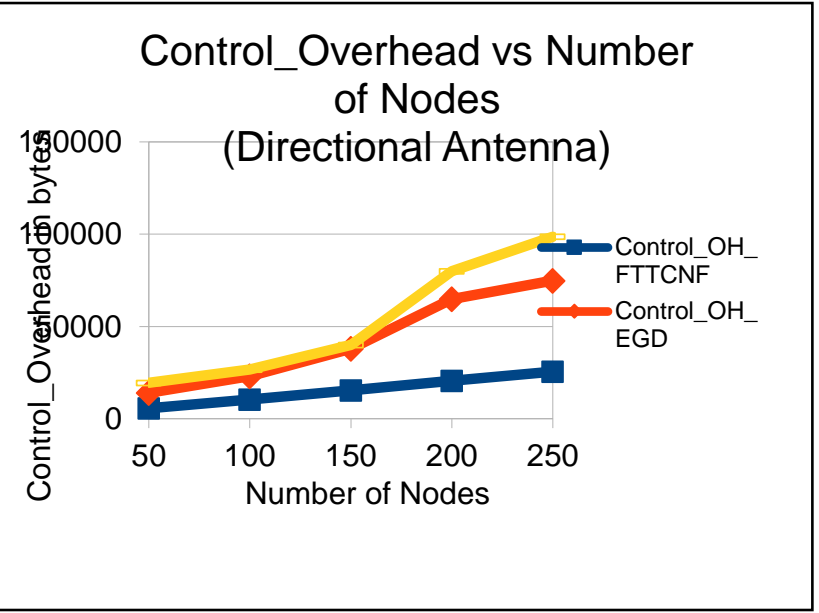

Fig 6 a) Control overhead vs Number of Nodes

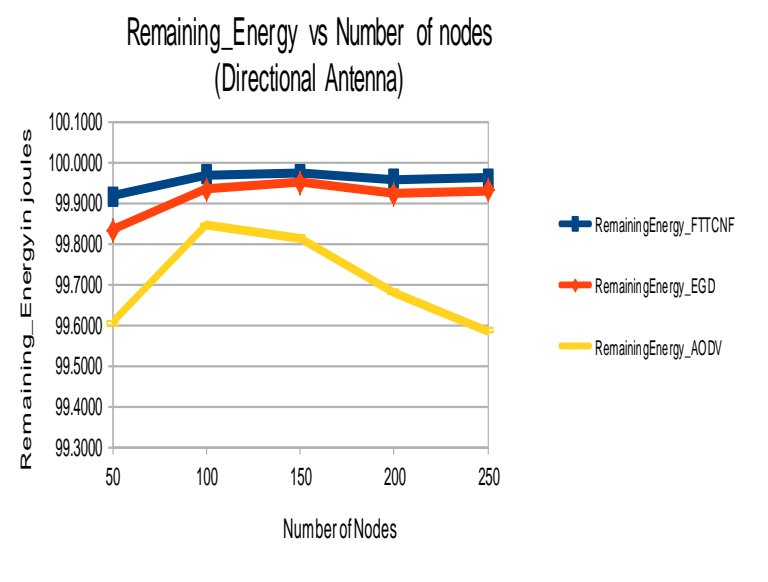

Fig 6 b) Remaining energy vs Number of Nodes

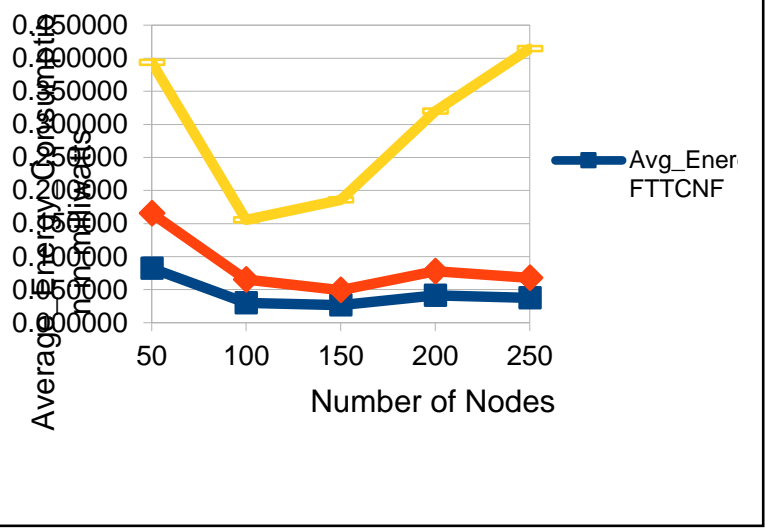

Fig 6 c) Average energy consumption vs Number of Nodes

In case of omni-directional antenna, overhead of FTTCNF is $61.27 \%$ less than EGD and $67.89 \%$ less than AODV. It is observed that control overhead of FTTCNF $0.72 \%$ greater than for directional when compared with omni-directional. FTTCNF and EGD almost perform the same in case of residual energy and FTTCNF has $0.02 \%$ residual energy greater than that of EGD and $0.12 \%$ greater than AODV as shown in Table 4 and Figures 7.a) to 7.c). This decrease in residual energy in AODV is due to the energy consumption for redundant broadcast of RREQ by nodes, retransmisions caused by packet loss and frequent re-route discoveries.

Table 4 Performance Evaluation FTTCNF, EGD \& AODV Dense Node distribution - OmniDirectional Antenna

\begin{tabular}{|l|l|l|l|}
\hline \multicolumn{2}{|c|}{$\begin{array}{l}\text { Control overhead } \\
\text { (in bytes) }\end{array}$} & $\begin{array}{l}\text { Delay } \\
\text { (in sec) }\end{array}$ & $\begin{array}{l}\text { Residual Energy } \\
\text { (in Joules) }\end{array}$ \\
\hline
\end{tabular}




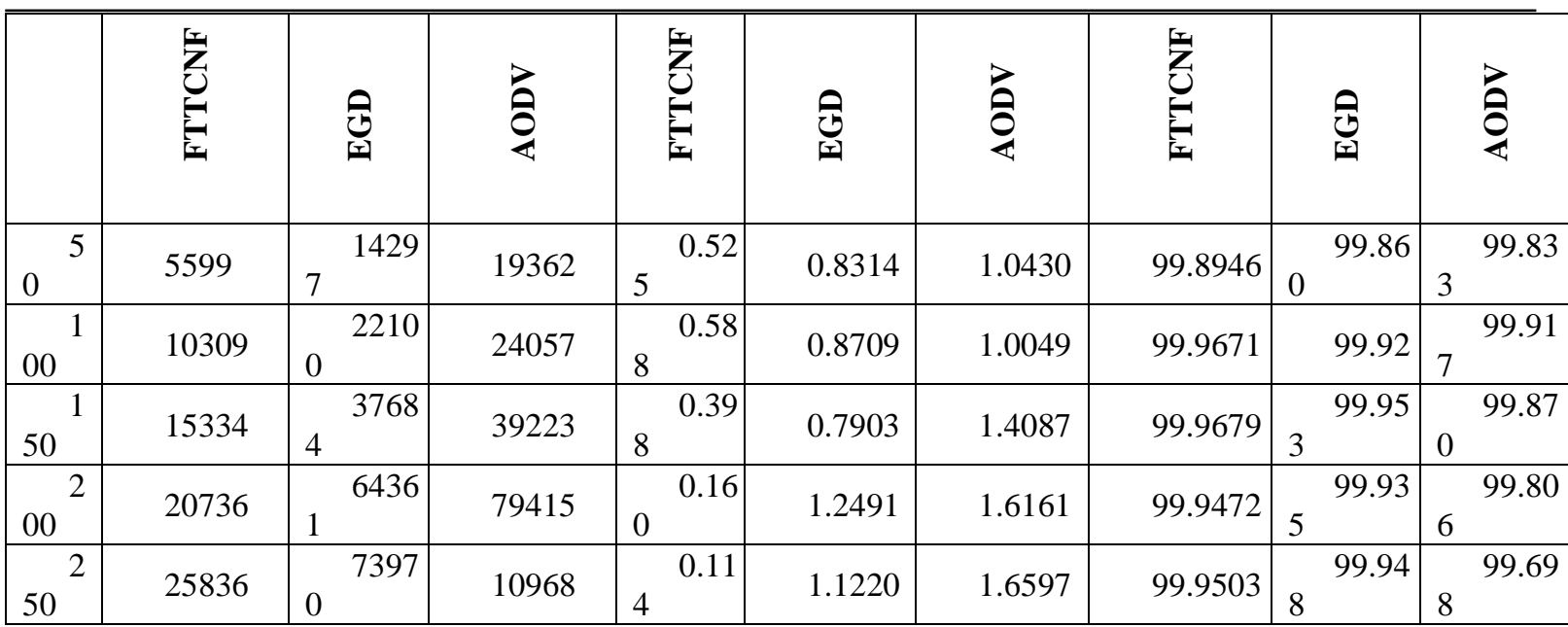

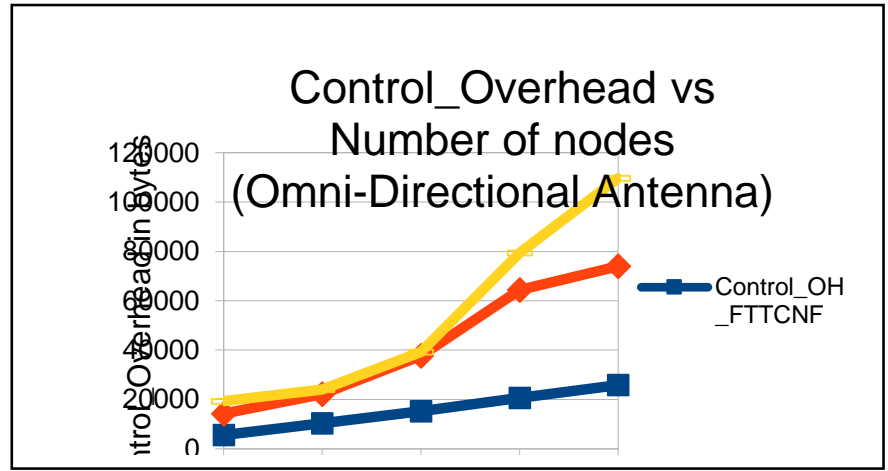

Figure 7.a) Control overhead vs Number of Nodes
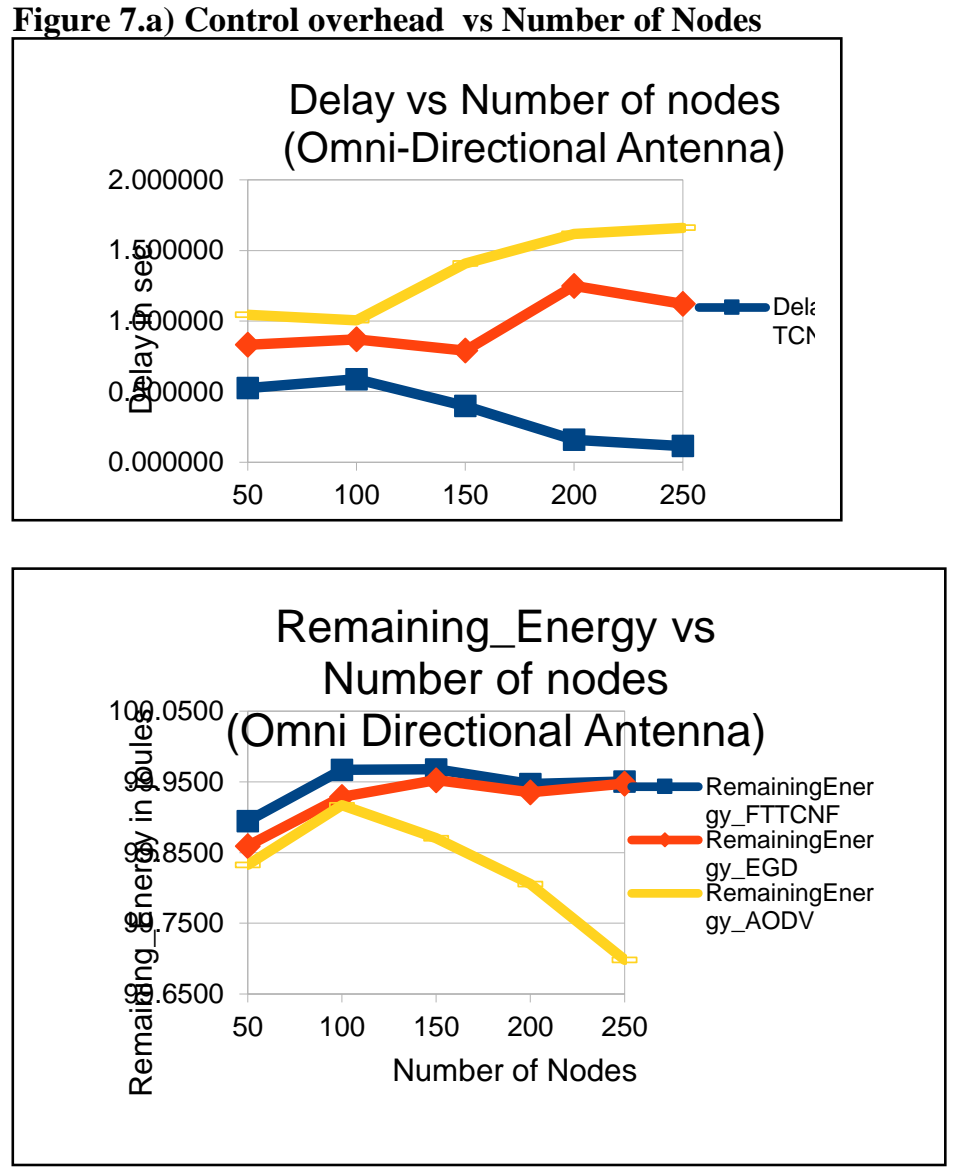

Figure 7.b) Delay vs Number of Nodes

Figure 7.c) Residual energy vs Number of Nodes

In case of Directional Antenna, an Average of $71.47 \%$ decrease in packet drop for FTTCNF when compared with EGD and it has an average decrease in packet drop 76.92\% when compared with AODV is observed. This performance degradation is due to the unstable route selection in AODV. Since FTTCNF avoids active 
participation of the nodes with energy less than that of threshold value in active routes by energy level monitoring scheme, packet drop due to node failures and link failures are significantly reduced. The performance of FTTCNF with respect to PDR, throughput and packet drop is as shown in Table 5.In throughput also FTTCNF shows better performance than EGD and AODV by an average increase of $11194.87 \mathrm{bps} 6437.22 \mathrm{bps}$ respectively. PDR of FTTCNF is $29.93 \%$ greater than EGD and $39.65 \%$ greater than AODV.

Table 5 Performance Evaluation FTTCNF, EGD \& AODV Directional Antenna

\begin{tabular}{|c|c|c|c|c|c|c|c|c|c|}
\hline \multirow[b]{2}{*}{$\begin{array}{l}\frac{n}{0} \\
\text { zo }\end{array}$} & \multicolumn{3}{|c|}{ Packet Drop Ratio (in \%) } & \multicolumn{3}{|c|}{ Throughput (in bps) } & \multicolumn{3}{|c|}{ PDR (in \%) } \\
\hline & $\sum_{\square}^{Z}$ & 司 & â & $\sum_{1}^{Z}$ & $\underset{6}{0}$ & $\stackrel{\partial}{a}$ & $\underbrace{Z}_{\text {U }}$ & అิ & $\stackrel{2}{a}$ \\
\hline 50 & 27.159 & 44.883 & 58.893 & 21900.1 & 6817.64 & 12841.8 & 72.8413 & 55.1167 & 41.107 \\
\hline 100 & 12.667 & 42.162 & 64.889 & 8962.85 & 2442.39 & 3744.14 & 87.3333 & 57.8378 & 35.111 \\
\hline 150 & 10.444 & 50.811 & 52.667 & 9190.91 & 2077.17 & 5047.48 & 89.5556 & 49.1892 & 47.333 \\
\hline 200 & 15.145 & 51.491 & 53.452 & 17263 & 4062.67 & 9839.65 & 84.8552 & 48.5095 & 46.548 \\
\hline 250 & 0.557 & 26.287 & 34.298 & 20230.8 & 6173.44 & 13888.5 & 99.4432 & 73.7127 & 65.702 \\
\hline
\end{tabular}

\subsubsection{Result analysis based on speed of nodes}

In this section, the performance of FTTCNF is analyzed by varying the speed of the nodes and keeping the number of nodes as 50 and the results are shown in Table 6 and Figures 8.a) to 8.c). It is observed that control overhead of FTTCNF is $58.78 \%$ less than that of EGD and $67.10 \%$ less than that of AODV for directional antenna. As FTTCNF finds the route by predicting link expire time based on RSSI, control overhead for reroute discovery, neighbour update is reduced significantly. The dropping ratio of FTTCNF is almost a straight line up to speed $3 \mathrm{~m} / \mathrm{s}$ and beyond that there is gradual increase in packet drop reaches a maximum value at $4 \mathrm{~m} / \mathrm{s}$ and finally reaches a minimum at $5 \mathrm{~m} / \mathrm{s}$. The dropping ratio is less than EGD by an average value $16.28 \%$ and $26 \%$ less than AODV. This is because of predicted link expire time in FTTCNF. Residual energy of FTTCNF is greater than EGD by $0.030 \%$ and $0.26 \%$ greater than AODV.

Table 6 Performance Evaluation FTTCNF, EGD,\& AODV-varying Speed Directional Antenna No. of nodes 50

\begin{tabular}{|c|c|c|c|c|c|c|c|c|c|}
\hline \multirow[b]{2}{*}{ 总 } & \multicolumn{3}{|c|}{ 莺 } & \multicolumn{3}{|c|}{ 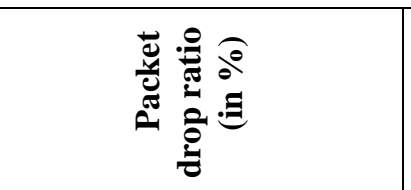 } & \multicolumn{3}{|c|}{ 焉 } \\
\hline & $\sum_{=1}^{Z}$ & છิ & 光 & $\sum_{1}^{Z}$ & $\underbrace{0}_{0=1}$ & 容 & $\sum_{1}^{Z}$ & Ð & 客 \\
\hline 1 & 5106 & 13197 & 18220 & 0.111 & 30 & 32.56 & 99.96 & 99.93 & 99.61 \\
\hline 2 & 5065 & 11850 & 14932 & 0.222 & 10.27 & 18.44 & 99.97 & 99.93 & 99.83 \\
\hline 3 & 5138 & 12879 & 15664 & 0.444 & 2.973 & 14.67 & 99.95 & 99.91 & 99.62 \\
\hline 4 & 5757 & 14020 & 19474 & 28.41 & 44.88 & 58.89 & 99.92 & 99.90 & 99.61 \\
\hline 5 & 5260 & 11759 & 13084 & 4 & 26.49 & 38.67 & 99.96 & 99.94 & 99.81 \\
\hline
\end{tabular}

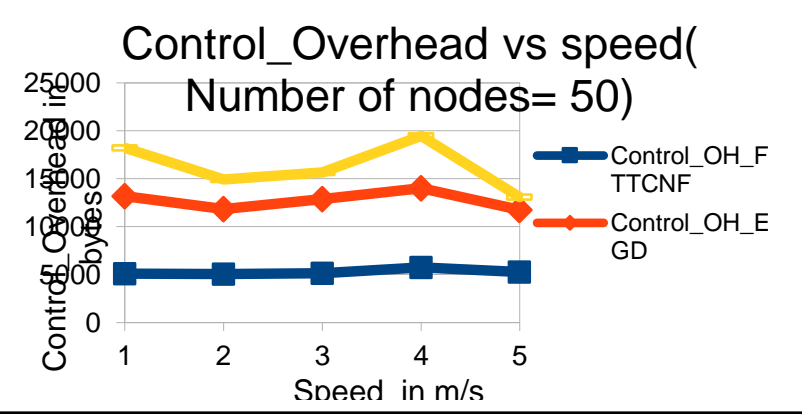

Figure 8.a) Control overhead vs Speed -Directional Antenna 


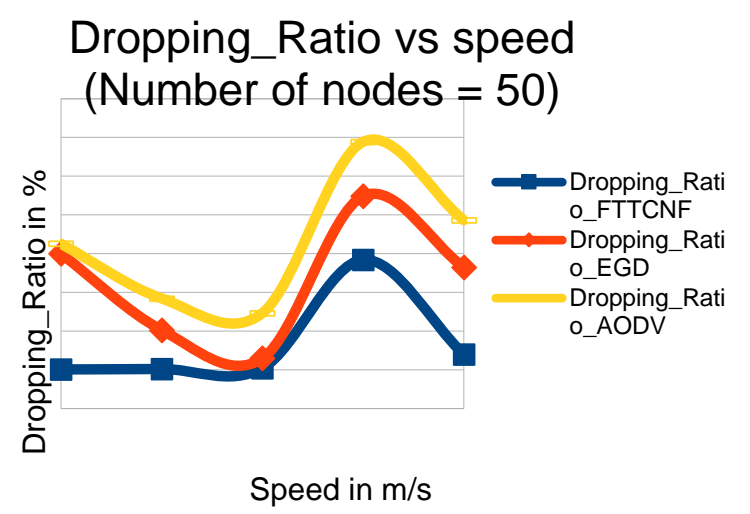

Figure 8.b) Dropping Ratio vs Speed -Directional Antenna

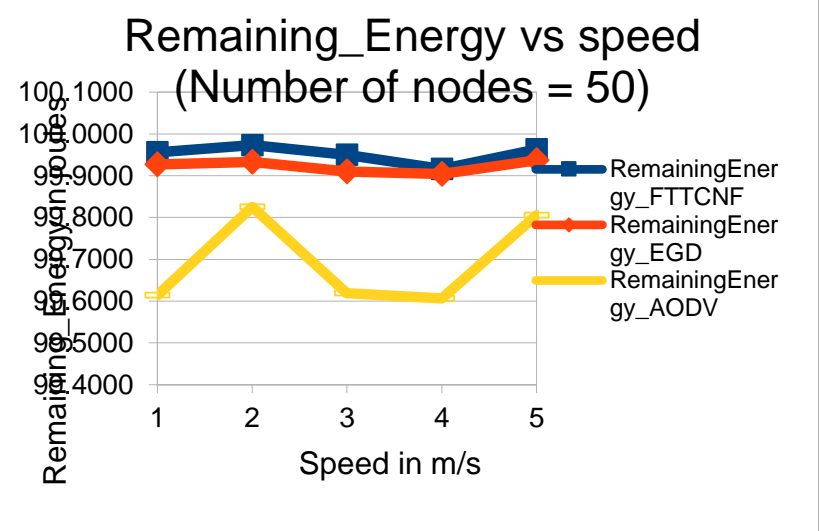

Figure 8. c) Residual Energy vs Speed -Directional Antenna

In case of omni- directional antenna, overhead of FTTCNF is 59.71\% less than EGD and $69.24 \%$ less than that of AODV. For omni-directional antenna, the packet drop ratio of FTTCNF is less than EGD by $11.38 \%$ and $19.37 \%$ less than AODV. Residual energy of FTTCNF is $0.043 \%$ greater than that of EGD and $0.29 \%$ greater than AODV. The results are shown in Table 7.The results for sparse distribution is as shown in Tables 8 and 9.

Table 7 Performance Evaluation FTTCNF, EGD,\& AODV-varying Speed Omni- Directional Antenna

\begin{tabular}{|c|c|c|c|c|c|c|c|c|c|}
\hline \multirow[b]{2}{*}{ 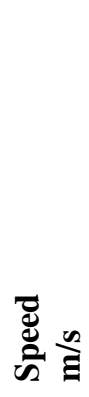 } & \multicolumn{3}{|c|}{ 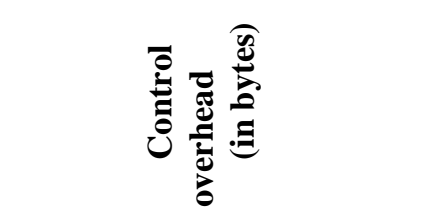 } & \multicolumn{2}{|c|}{ 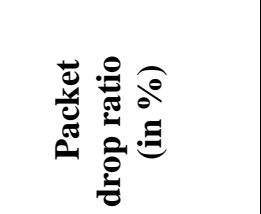 } & \multicolumn{4}{|c|}{ 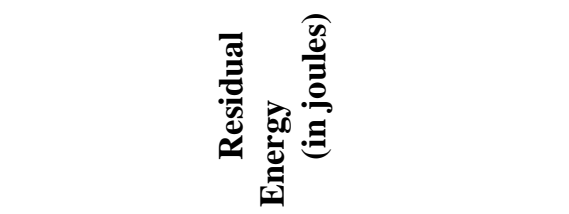 } \\
\hline & 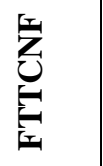 & 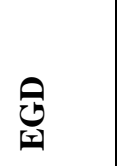 & 常 & 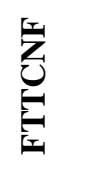 & ఫ్తి & 官 & 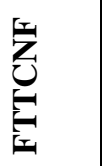 & 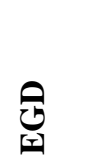 & $\frac{2}{2}$ \\
\hline 1 & 5020 & 13259 & 18220 & 0.111 & 39.19 & 40.22 & 99.92 & 99.90 & 99.92 \\
\hline 2 & 5042 & 12449 & 14932 & 0.444 & 9.73 & 16.22 & 99.96 & 99.92 & 99.96 \\
\hline 3 & 5152 & 12753 & 15664 & 0.333 & 8.38 & 41.67 & 99.97 & 99.92 & 99.97 \\
\hline 4 & 5599 & 14297 & 19474 & 22.29 & 46.50 & 69.67 & 99.93 & 99.89 & 99.93 \\
\hline 5 & 5192 & 11927 & 13084 & 3.78 & 30.27 & 33.78 & 99.95 & 99.91 & 99.95 \\
\hline
\end{tabular}

Table 8 Performance of FTTCNF (Sparse Node)- Directional Antenna 


\begin{tabular}{|c|c|c|c|c|c|}
\hline 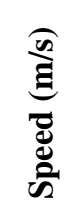 & 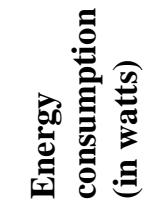 & 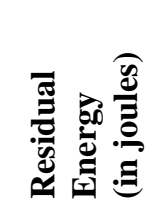 & 㔯 & 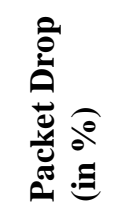 & 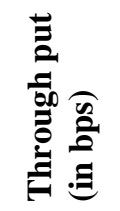 \\
\hline 1 & 0.04350 & 99.9565 & 96.9963 & 3.0037 & 29377.6 \\
\hline 2 & 0.03170 & 99.9683 & 98.7573 & 1.2427 & 29655.4 \\
\hline 3 & 0.04110 & 99.9589 & 85.4945 & 14.5055 & 17356.7 \\
\hline 4 & 0.06269 & 99.9373 & 97.3529 & 2.6471 & 29537.6 \\
\hline 5 & 0.07046 & 99.9295 & 90.3297 & 9.6703 & 18338.3 \\
\hline
\end{tabular}

Table 9 Performance of FTTCNF (Sparse Node) - Omni-Directional Antenna

\begin{tabular}{|c|c|c|c|c|c|}
\hline 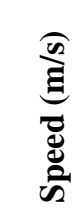 & 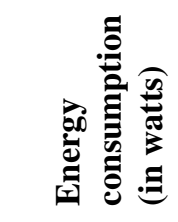 & 焉 & 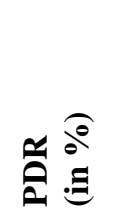 & 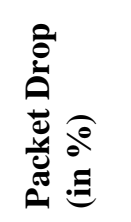 & 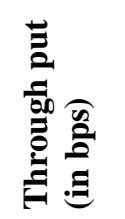 \\
\hline 1 & 0.07623 & 99.9238 & 96.9231 & 3.0769 & 29355.4 \\
\hline 2 & 0.04106 & 99.9589 & 94.5055 & 5.4945 & 19186.1 \\
\hline 3 & 0.03364 & 99.9664 & 93.956 & 6.0440 & 19074.5 \\
\hline 4 & 0.06470 & 99.9353 & 97.7206 & 2.2794 & 29649.2 \\
\hline 5 & 0.04675 & 99.9532 & 89.7802 & 10.2198 & 18226.8 \\
\hline
\end{tabular}

4.4.3 Result analysis based on traffic pattern

In this section, the performance analysis of FTTCNF was done by varying data rate separately for both directional and omni-directional antenna. The comparative analysis for FTTCNF, EGD and AODV were shown in Tables 10 to 12 for directional antenna and 13 to 15 for omni-directional antenna. In directional antenna AODV performed better w.r.t control overhead compared with EGD. When varying the data rate EGD showed negative effect w.r.t control overhead, PDR and packet drop. AODV also showed negative effect for PDR and packet drop. Although FTTCNF showed reduced performance when compared with other scenarios, it outperformed than AODV and EGD. This performance of FTTCNF is achieved by finding the stable path by the prediction of link expiration and avoidance of expire nodes in the path calculation. For the same scenario with omni- directional antenna the performance of AODV and EGD are slightly improved. PDR of FTTCNF was increased by nearly $2 \%$, control overhead by $0.2 \%$ and throughput by nearly $30 \mathrm{bps}$ for omni-directional antenna and packet drop was decreased by $3 \%$.

Table 10 Performance of FTTCNF Varying Data Rate -Directional Antenna

\begin{tabular}{|c|c|c|c|c|c|c|}
\hline \multicolumn{7}{|c|}{ FTTCNF- Varying Data Rate - Directional Antenna } \\
\hline 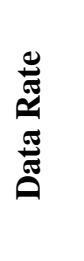 & 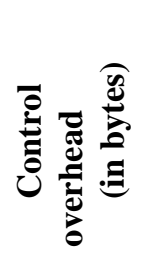 & 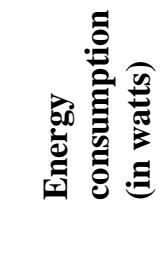 & 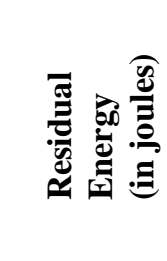 & 余 & 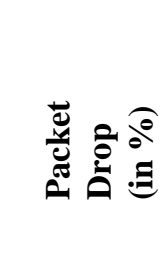 & 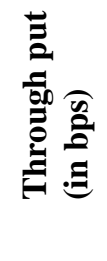 \\
\hline 0.4 & 10130 & 0.008658 & 99.9913 & 72.22 & 27.7778 & 305.88 \\
\hline 0.5 & 10109 & 0.009087 & 99.9909 & 90.91 & 9.0909 & 476.19 \\
\hline 0.6 & 10195 & 0.009692 & 99.9903 & 92.59 & 7.4074 & 576.92 \\
\hline 0.7 & 10175 & 0.010185 & 99.9898 & 90.32 & 9.6774 & 653.33 \\
\hline 0.8 & 10060 & 0.009018 & 99.9910 & 55.56 & 44.4444 & 457.14 \\
\hline
\end{tabular}

Table 11 Performance of EGD Varying Data Rate -Directional Antenna

EGD - Varying Data Rate - Directional Antenna 


\begin{tabular}{|c|c|c|c|c|c|c|}
\hline 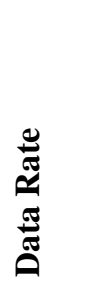 & 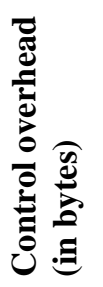 & 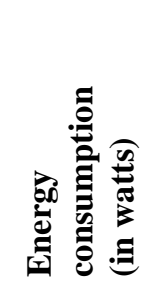 & 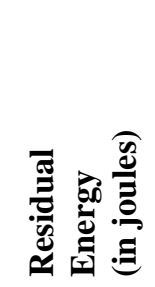 & $\widehat{a}$ & 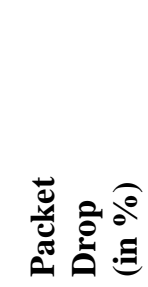 & 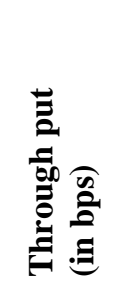 \\
\hline 0.4 & 21437 & 0.0112 & 99.9888 & 55.56 & 44.44 & 235.29 \\
\hline 0.5 & 21766 & 0.0116 & 99.9884 & 65.23 & 34.78 & 340.91 \\
\hline 0.6 & 21322 & 0.0112 & 99.9888 & 55.56 & 44.44 & 346.15 \\
\hline 0.7 & 21841 & 0.0116 & 99.9884 & 51.61 & 48.39 & 373.33 \\
\hline 0.8 & 22080 & 0.0118 & 99.9882 & 50 & 50. & 411.43 \\
\hline
\end{tabular}

Table 12 Performance of AODV Varying Data Rate -Directional Antenna

\begin{tabular}{|c|c|c|c|c|c|c|}
\hline \multicolumn{7}{|c|}{ AODV- Varying Data Rate - Directional Antenna } \\
\hline 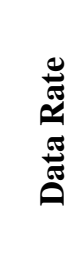 & 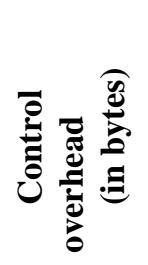 & 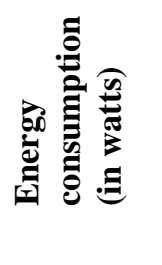 & 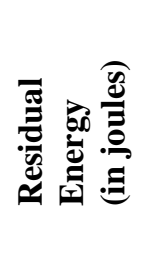 & 总 & 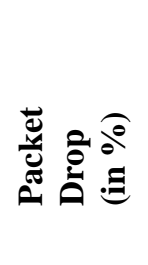 & 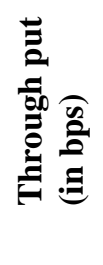 \\
\hline 0.4 & 12339 & 0.013645 & 99.9864 & 18.182 & 81.8182 & 83.125 \\
\hline 0.5 & 12245 & 0.013375 & 99.9866 & 15.385 & 84.6154 & 86.589 \\
\hline 0.6 & 12436 & 0.014232 & 99.9858 & 18.75 & 81.2500 & 124.69 \\
\hline 0.7 & 12441 & 0.014685 & 99.9853 & 15 & 85.0000 & 115.13 \\
\hline 0.8 & 12350 & 0.013778 & 99.9862 & 9.5238 & 90.4762 & 83.125 \\
\hline
\end{tabular}

Table 13 Performance of FTTCNF Varying Data Rate (Omni-Directional)

\begin{tabular}{|c|c|c|c|c|c|c|}
\hline 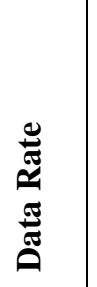 & 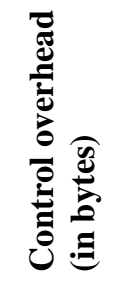 & 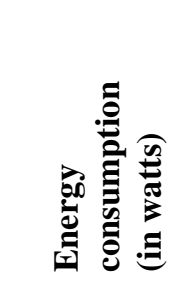 & 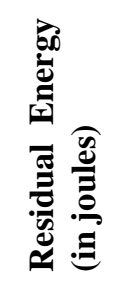 & $\begin{array}{l}\widehat{o} \\
\Xi \\
\Xi \\
a\end{array}$ & 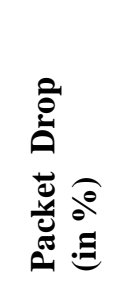 & 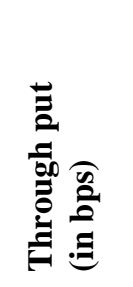 \\
\hline 0.4 & 10108 & 0.008412 & 99.9914 & 52.0000 & 49.0000 & 211.765 \\
\hline 0.5 & 10124 & 0.009574 & 99.9905 & 90.9091 & 9.0909 & 476.19 \\
\hline 0.6 & 10123 & 0.010167 & 99.9902 & 88.8889 & 11.1111 & 553.846 \\
\hline 0.7 & 10124 & 0.010324 & 99.9904 & 70.9677 & 29.0323 & 513.333 \\
\hline 0.8 & 10052 & 0.010435 & 99.9894 & 83.3333 & 16.6667 & 685.714 \\
\hline
\end{tabular}

Table 14 Performance of EGD Varying Data Rate (Omni Directional)

\begin{tabular}{|c|c|c|c|c|c|c|}
\hline 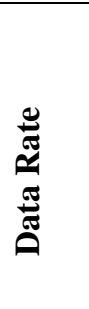 & 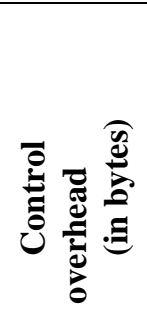 & 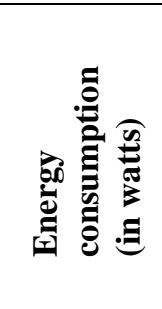 & 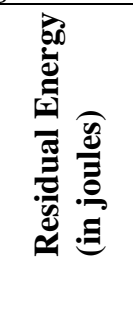 & 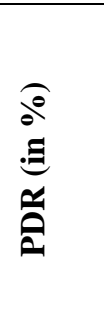 & 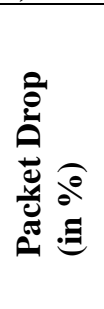 & 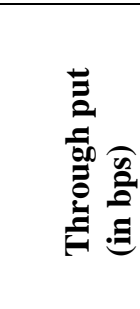 \\
\hline 0.4 & 21260 & 0.011419 & 99.9502 & 47.56 & 54.44 & 135.294 \\
\hline 0.5 & 21701 & 0.011713 & 99.9471 & 50.00 & 50.00 & 261.905 \\
\hline 0.6 & 21749 & 0.011912 & 99.9466 & 51.85 & 48.15 & 323.077 \\
\hline 0.7 & 21889 & 0.012028 & 99.9459 & 51.61 & 48.39 & 373.333 \\
\hline 0.8 & 22091 & 0.012219 & 99.9437 & 52.78 & 47.22 & 434.286 \\
\hline
\end{tabular}




\begin{tabular}{l} 
Table 15 \\
\hline
\end{tabular}

\section{Conclusion}

This Proposed protocol is a modified form of the base code of AODV implemented using hybrid neuro fuzzy technique in Network Simulator NS-2. FTTCNF protocol broadcasts one hop Hello packets to find the neighbours in sequence. To construct the complete list of neighbours RSSI value was computed to find the neighbours from which a powerful signal was received. LET was also computed to know the link disconnection period to get accurate PSF. To extend the network lifetime, each node computes and compares its current energy level with given energy threshold. If it is lower than given energy threshold then the node itself rejects forwarding RREQ. Thus the goal was to restrict the lower energy node to participate in the active routing path. In addition, using neuro fuzzy computation complex problem of path selection was solved. FTTCNF constructs linguistic rules building a fuzzy inference set. Since FTTCNF finds a stable route, the life time of the network, throughput and the average residual energy were all improved on comparison with EGD and AODV. The performance analysis showed that in all scenarios by using directional and omni-directional antenna FTTCNF performed well.

\section{References}

N. Hosseinzadeh, Member, IEEE, A. Kalam, "A Rule-Based Fuzzy Power System Stabilizer Tuned by a Neural Network" IEEE Transactions on Energy Conversion, Vol. 14, No. 3, September 1999.

Sushmita Mitra, Member, IEEE, and Yoichi Hayashi, Senior Member, IEEE, "Neuro-Fuzzy Rule Generation: Survey in Soft Computing Framework" IEEE TRANSACTIONS ON NEURAL NETWORKS, VOL. 11, NO. 3, MAY 2000.

Robert Fuller Eotvos Lorand University, Budapest, "Neuro-Fuzzy Methods for Modelling and Fault DiagnosisLisbon, August 31 and September 1, 2001.

Romit Roy Choudhury, Xue Yang, Ram Ramanathan and, Nitin H. Vaidya, "Using Directional Antennas for Medium Access Control in Ad Hoc Networks" MOBICOM'02, Atlanta, Georgia, USA September 23-28-2002.

Johann Lopez, Jose M. Barcelo, Jorge Garcia Vidal, “Analyzing The Overhead in Mobile Ad-hoc Network with A Hierarchical Routing Structure, 2004.

Alaa Muqattash, Student Member, IEEE, and Marwan M. Krunz, Member, IEEE, "A Distributed Transmission Power Control Protocol for Mobile Ad Hoc Networks

” IEEE TRANSACTIONS ON MOBILE COMPUTING, VOL. 3, NO. 2, APRIL-JUNE 2004.

R. Chellappa Doss, A. Jennings, N. Shenoy, “A Review of Current Mobility Prediction Techniques For Ad hoc Networks "Proceedings of the $4^{\text {th }}$ IASTED International Multi-Conference Wireless and Optical Communication, Banff, Canada July 8-10,2004.

Heikki Koiovo, David S, A.P. Paplinski, "Basic concepts of Neural Networks and Fuzzy Logic Systems "May 25, 2005.

Jie Wu, Senior Member, IEEE, and Fei Dai, Member, IEEE, "Mobility-Sensitive Topology Control in Mobile Ad Hoc Networks

" IEEE TRANSACTIONS ON PARALLEL AND DISTRIBUTED SYSTEMS, VOL. 17, NO. 6, JUNE 2006.

Basel Alawieh, Student Member, IEEE, Chadi M. Assi, Member, IEEE, and Wessam Ajib, Member, IEEE, "Distributed Correlative Power Control Schemes for

Mobile Ad hoc Networks Using Directional Antennas

” IEEE TRANSACTIONS ON VEHICULAR TECHNOLOGY, VOL. 57, NO. 3, MAY 2008.

Saleh Ali K.Al-Omari, Putra Sumari, "An Overview of Mobile Adhoc Networks for The Existing Protocols and Applications" International journal on applications of graph theory in wireless ad hoc networks and sensor networks (Graph-Hoc), Vol.2, No.1, March 20210.

Arash Dana Mohamad Hadi Babaei, “A Fuzzy Based Stable Routing Algorithm for MANET” IJCSI International Journal of Computer Science Issues, Vol. 8, Issue 1, January 2011. 
Natarajan Meghanathan, “A Unicast Stable Path Routing Protocol for Mobile Ad hoc Networks based on the Inverse of Link Expiration Time "Network Protocols and Algorithms, ISSN 1943-3581, Vol. 3, No. 3, 2011.

Xin Ming Zhang, Member, IEEE, En Bo Wang, Jing Jing Xia, and Dan Keun Sung, Senior Member, IEEE, "An Estimated Distance-Based Routing

Protocol for Mobile Ad hoc Networks

" IEEE TRANSACTIONS ON VEHICULAR TECHNOLOGY, VOL. 60, NO. 7, SEPTEMBER 2011.

Andy An-Kai Jeng and Rong-Hong Jan, Senior Member, IEEE Computer Society, "Adaptive Topology Control for Mobile Ad Hoc Networks" IEEE TRANSACTIONS ON PARALLEL AND DISTRIBUTED SYSTEMS, VOL. 22, NO. 12, DECEMBER 2011.

Hiroki Nishiyama, Member, IEEE, Thuan Ngo, Nirwan Ansari, Fellow, IEEE, and Nei Kato, Senior Member, IEEE, "On Minimizing the Impact of Mobility on

Topology Control in Mobile Ad Hoc Networks

" IEEE TRANSACTIONS ON WIRELESS COMMUNICATIONS, VOL. 11, NO. 3, MARCH 2012.

Pinki Nayak, Rekha Agrawal, and Seema Verma, "Energy Aware Routing Scheme for Mobile Ad hoc Network Using Variable Range Transmission "International Journal of Ad hoc, Sensor \& Ubiquitous Computing (IJASUC) Vol.3, No.4, August 2012.

Ramezanali Sadeghzadeh, Neshat Varjavand Naseri and Afsane Saee, "A Survey of Using Directional Antennas in Ad Hoc Networks" International Journal on AdHoc Networking Systems (IJANS) Vol. 2, No. 4, October 2012.

Erkan Kayacan, Student Member, IEEE, Erdal Kayacan, Senior Member, IEEE, Herman Ramon, and Wouter Saeys, "Adaptive Neuro-Fuzzy Control of a Spherical Rolling Robot Using Sliding-Mode-Control-Theory-Based Online Learning Algorithm” IEEE TRANSACTIONS ON CYBERNETICS, VOL. 43, NO. 1, FEBRUARY 2013.

Israat Tanzeena Haque, Member, IEEE, “On the Overheads of Ad Hoc Routing Schemes" IEEE SYSTEMS JOURNAL, VOL. 9, NO. 2, JUNE 2015.

Gopal Singh, Deepak Saini, Rahul Rishi, Harish Rohil, "Role of Link expiration time to make reliable link between the nodes in MANETs: A Review "International Journal of Applied Engineering Research ISSN 09734562 Volume 11, Number 72016.

Stefano Rosati, Member, IEEE, Karol Kruzelecki, IEEE, Grégoire Heitz, Dario Floreano, Senior Member, IEEE, and Bixio Rimoldi, Fellow, IEEE, "Dynamic Routing for Flying Ad Hoc Networks" IEEE TRANSACTIONS ON VEHICULAR TECHNOLOGY, VOL. 65, NO. 3, MARCH 2016.

K. Sakthidasan Sankaran, N. Vasudevan, K. R. Devabalaji, T. S. Babu, H. H. Alhelou and T. Yuvaraj, "A Recurrent Reward Based Learning Technique for Secure Neighbor Selection in Mobile AD-HOC Networks," in IEEE Access, doi: 10.1109/ACCESS.2021.3055422.

Damilare Oluwole Akande And Mohd Fadzli Mohd Salleh , (Member, IEEE) "A Network Lifetime Extension-Aware Cooperative MAC Protocol for MANETs With Optimized Power Control", IEEE Access,VOL.7 pp.18546 - 18557.

B. H. Khudayer, M. Anbar, S. M. Hanshi and T. Wan, "Efficient Route Discovery and Link Failure Detection Mechanisms for Source Routing Protocol in Mobile Ad-Hoc Networks," in IEEE Access, vol. 8, pp. 2401924032, 2020, doi: 10.1109/ACCESS.2020.2970279.

A. Bhardwaj and H. El-Ocla, "Multipath Routing Protocol Using Genetic Algorithm in Mobile Ad Hoc Networks," in IEEE Access, vol. 8, pp. 177534-177548, 2020, doi: 10.1109/ACCESS.2020.3027043. 